Special issue, XLVI Extraordinary Congress of Polish Physicists, Warsaw, Poland, October 16-18, 2020

\title{
Relativistic Relative Velocities and Relativistic Acceleration
}

\author{
G.M. KoCZAN* \\ Warsaw University of Life Sciences (WULS/SGGW), Warsaw, Poland \\ Doi: 10.12693/APhysPolA.139.401 \\ *e-mail: grzegorz_koczan@sggw.edu.pl

\begin{abstract}
It turns out that the standard application of the four-vector SR formalism does not include the concept of relative velocity. Only the absolute velocity is described by the four-vector, and even the Lorentz transformation parameters are described by the three-dimensional velocity. This gap in the development of the SR formalism reflects the lack of some significant velocity subtraction operations. The differential application of these operations leads to a relativistic acceleration.
\end{abstract}

topics: velocity subtraction, 3D and 4D relative velocity, differential of velocity, relativistic acceleration

\section{Introduction}

Relative velocity is the defined difference of two velocities. In the theory of relativity, such a difference is most easily realized using the Lorentz transformation [1]:

$$
\begin{aligned}
& x^{\prime}=\gamma_{v}(x-v t), \quad t^{\prime}=\gamma_{v}\left(t-\frac{v}{c^{2}} x\right), \\
& y^{\prime}=y, \quad z^{\prime}=z,
\end{aligned}
$$

where $\gamma_{v}=1 / \sqrt{1-v^{2} / c^{2}}$. The Lorentz transformation of velocity is some way of subtracting velocities

$$
\begin{aligned}
& u_{x}^{\prime}=\frac{\mathrm{d} x^{\prime}}{\mathrm{d} t^{\prime}}=\frac{u_{x}-v}{1-u_{x} v / c^{2}}=: u_{x} \ominus v, \\
& u_{y}^{\prime}=\frac{\mathrm{d} y^{\prime}}{\mathrm{d} t^{\prime}}=u_{y} \frac{\sqrt{1-v^{2} / c^{2}}}{1-u_{x} v / c^{2}}=: u_{y} \boxminus_{u_{x}} v .
\end{aligned}
$$

The component parallel to the boost velocity is subject to the $\ominus$ subtraction, and the perpendicular components are subject to the $\boxminus_{u_{x}}$ operation, which is parameter dependent and is more like multiplication than subtraction. If we apply appropriate operations for all components at the same time $\left(\ominus, \boxminus_{u_{x}}, \boxminus_{u_{x}}\right)$ then we will get the full vector subtraction $\ominus_{0}$ of the Einstein velocity [2]:

$$
\boldsymbol{u}^{\prime}=\frac{\boldsymbol{u}-\boldsymbol{v}+\frac{\gamma_{v}}{\gamma_{v}+1}(\boldsymbol{u} \times \boldsymbol{v}) \times \boldsymbol{v} / c^{2}}{1-\boldsymbol{u} \boldsymbol{v} / c^{2}}=: \boldsymbol{u} \ominus_{0} \boldsymbol{v} .
$$

Einstein, like many behind him (but not all [3, 4]), considered addition, not subtraction. However, the addition here is less natural and leads to an unequally ambiguous sequence $\boldsymbol{u} \oplus \boldsymbol{v}=\boldsymbol{u} \ominus_{0}(-\boldsymbol{v})$ vs. $\quad \boldsymbol{u} \oplus \boldsymbol{v}=\boldsymbol{v} \ominus_{0}(-\boldsymbol{u})$ or $\quad \boldsymbol{v} \oplus \boldsymbol{u}=\boldsymbol{u} \ominus_{0}(-\boldsymbol{v})$. The latter convention is most often used [5, 6], which changes the right-hand character of the operation to the left-hand one.
It is also worth calculating the velocity differential in relation to the velocity subject to the boost (compare [7]):

$$
\begin{aligned}
\mathrm{d} u_{x}^{\prime} & :=\left.\frac{\partial u_{x}^{\prime}}{\partial u_{x}}\right|_{u_{x}=v} \mathrm{~d} u_{x}=\frac{\mathrm{d} u_{x}}{1-v^{2} / c^{2}}=\gamma_{v}^{2} \mathrm{~d} u_{x} \\
\mathrm{~d} u_{y}^{\prime} & :=\left.\frac{\partial u_{y}^{\prime}}{\partial u_{x}}\right|_{u_{y}=0} \mathrm{~d} u_{x}+\left.\frac{\partial u_{y}^{\prime}}{\partial u_{y}}\right|_{u_{x}=v} \mathrm{~d} u_{y}=\gamma_{v} \mathrm{~d} u_{y} .
\end{aligned}
$$

In vector terms, this equation takes the form [8]:

$$
\begin{gathered}
\mathrm{d} \boldsymbol{u}^{\prime}:=\left(\mathrm{d} \boldsymbol{u}^{\prime}\right)_{\boldsymbol{v}}:=\left.\frac{\partial \boldsymbol{u}^{\prime}}{\partial u_{i}}\right|_{\boldsymbol{u}=\boldsymbol{v}} \mathrm{d} u_{i}= \\
\gamma_{v} \mathrm{~d} \boldsymbol{u}+\frac{\gamma_{v}^{3}}{\gamma_{v}+1} \frac{\boldsymbol{v}(\boldsymbol{v} \mathrm{d} \boldsymbol{u})}{c^{2}} .
\end{gathered}
$$

It turns out that the differential calculated with respect to the second variable is basically only different in sign

$$
\begin{aligned}
\left(\mathrm{d} \boldsymbol{u}^{\prime}\right)_{\boldsymbol{u}} & :=\left.\frac{\partial \boldsymbol{u}^{\prime}}{\partial v_{i}}\right|_{\boldsymbol{v}=\boldsymbol{u}} \mathrm{d} v_{i}= \\
-\gamma_{u} \mathrm{~d} \boldsymbol{v} & -\frac{\gamma_{u}^{3}}{\gamma_{u}+1} \frac{\boldsymbol{u}(\boldsymbol{u} \mathrm{d} \boldsymbol{v})}{c^{2}} .
\end{aligned}
$$

At high speeds, it is natural that the $\mathrm{d} u_{x}$ differential, which is also the differential of $u$ value, scales as in (5) with the appropriate power of the gamma factor [9]. Such scaling is necessary so that the transformed speed value does not exceed the speed of light. On the other hand, other scaling of the perpendicular speed differential (6) results from time dilation between transformed systems. In [10] an attempt was made to reconcile the scaling of both differentials. However, the scaling of the perpendicular differential does not follow the idea of an essentially directional (axial) change in the velocity vector - an analogous discrepancy is included in $(3)$. 
Another disadvantage of the Einstein subtraction (4) is the lack of its antisymmetry and the lack of associativity for the addition cooperation $[6,11,12]$. As we will see, with the right approach to the Lorentz boosts, the problem of the lack of velocity subtraction antisymmetry can be eliminated. In a sense, the problem of lack of antisymmetry also does not occur at the differential level (7) and (8). On the other hand, the problem of the lack of velocity addition associativity is more serious and is not covered by this paper. This problem has been tackled in [12-14]. It should be emphasized that the lack of associativity explicitly concerns the velocity composition, not the Lorentz group.

Although the four-vector approach is not considered in the SR for the velocity subtraction, let us consider such four-velocities [15, 16]:

$$
\mu^{\mu}=\left(\gamma_{u} c, \gamma_{u} \boldsymbol{u}\right), \quad \nu^{\mu}=\left(\gamma_{v} c, \gamma_{v} \boldsymbol{v}\right) .
$$

Let us try to provisionally write (2) and (3) by subtracting four-velocities

$$
\frac{\mu^{\mu}-\nu^{\mu}}{\mu \circ \nu / c^{2}}=\frac{\left(\gamma_{u} c-\gamma_{v} c, \gamma_{u} \boldsymbol{u}-\gamma_{v} \boldsymbol{v}\right)}{\gamma_{u} \gamma_{v}\left(1-\boldsymbol{u v} / c^{2}\right)},
$$

where $\mu \circ \nu=\mu_{\beta} \nu^{\beta}$ is the scalar product of fourvectors. Unfortunately, this formula is not valid for the parallel component (2), although it is correct for the perpendicular component (3). Nevertheless, it turns out that (4) can be formally converted into a four-vector form with the appropriate tools (see [17, 18]). However, this paper focuses on other (as it will turn out to be valid) methods of velocity subtraction that are ideologically similar to $(10)$.

\section{Axial subtraction of velocities}

As indicated above, the orthodox velocity subtraction method (4) has several natural disadvantages. The most artificial is the operation of $\boxminus_{u_{x}}$ for components perpendicular to the subtrahend velocity, which differs significantly from the operation of $\ominus$ for parallel components. Therefore, we can try to standardize the operation of $\ominus$ for all components [5, 19]:

$$
\begin{aligned}
& w_{\mid x}:=u_{x} \ominus v=\frac{u_{x}-v}{1-u_{x} v / c^{2}}, \\
& w_{\mid y}:=u_{y} \ominus v_{y}=u_{y} \ominus 0=u_{y}, \\
& w_{\mid z}:=u_{z} .
\end{aligned}
$$

If the $x$ axis goes along $\boldsymbol{v}$, then the above equations define an equivalent vector operation $\ominus_{\|}=$ $(\ominus, \ominus, \ominus)$ :

$$
\boldsymbol{u} \ominus_{\|} \boldsymbol{v}:=\frac{\boldsymbol{u}-\boldsymbol{v}+\frac{\boldsymbol{u} \boldsymbol{v}}{v^{2}}(\boldsymbol{u} \times \boldsymbol{v}) \times \boldsymbol{v} / c^{2}}{1-\boldsymbol{u v} / c^{2}}=: \boldsymbol{w}_{\mid}
$$

This subtraction only changes the parallel component to the velocity subtrahend, so it will be called axial subtraction and $\boldsymbol{w}_{\mid}$relative axial velocity.
Statement 1. Axial subtraction of velocity vectors (14) is of the form

$$
\boldsymbol{u} \ominus_{\|} \boldsymbol{v}=\boldsymbol{w}_{\mid}=\boldsymbol{u}-\varphi \boldsymbol{v},
$$

where the $\varphi$ function results from parallel subtraction (11).

Proof. Using (11) to (15) allows to calculate

$$
\varphi=\frac{1-u_{x}^{2} / c^{2}}{1-u_{x} v / c^{2}}=\frac{1-(\boldsymbol{u} \boldsymbol{v})^{2} /(c v)^{2}}{1-\boldsymbol{u v} / c^{2}},
$$

which inserted into (15) after simplifications leads to axial subtraction (14), Q.E.D.

Calculating the differentials of relative axial velocity is not difficult

$$
\begin{aligned}
& \mathrm{d} w_{\mid x}=\gamma_{v}^{2} \mathrm{~d} u_{x}, \quad \mathrm{~d} w_{\mid y}=\mathrm{d} u_{y}, \\
& \mathrm{~d} w_{\mid z}=\mathrm{d} u_{z} .
\end{aligned}
$$

This form of differentials (which will be confirmed later) is more general than that of (5) and (6). The perpendicular part of the differential (6) is modified here in a similar way as in the paper [20].

\section{Binary subtraction of $4 \mathrm{D}$ and $3 \mathrm{D}$ velocities}

Most often, an ordinary operation is a binary operation. Some velocity operations depend on additional parameters, for example operation (3) or ternary subtraction, discussed in the next section. In this article, the terms "binary" and "ternary" have the nature of proper names borrowed from Oziewicz [17, 18] — they are adequate in the case of $4 \mathrm{D}$, while in $3 \mathrm{D}$ they are less precise.

Consider some generalization of the four-vector velocity subtraction (10) as a linear combination

$$
\omega^{\mu}:=(\mu \triangleleft \nu)^{\mu}:=\lambda_{2} \mu^{\mu}-\lambda_{1} \nu^{\mu} .
$$

In order to determine the $\lambda_{1}, \lambda_{2}$ form factors, we impose two conditions: orthogonality to the subtrahend four-velocity and reduction of the result to ordinary velocity for zero subtrahend three-velocity $\left(u_{i} \equiv u^{i}\right)$ :

$$
\omega \circ \nu:=0, \quad \omega^{i}(v=0):=u^{i}=\mu^{i} / \gamma_{u} .
$$

These conditions with the signature +--$\left(\mu_{i}=-\mu^{i}\right)$ lead to the value $\lambda_{1}=1, \lambda_{2}=c^{2} /(\mu \circ \nu)$ and the final form of the relative binary fourvelocity (see $[12,17,19,21])$ :

$$
\omega^{\mu}=(\mu \varangle \nu)^{\mu}=\frac{c^{2}}{\mu \circ \nu} \mu^{\mu}-\nu^{\mu} .
$$

Statement 2. The norm of the binary subtraction of the four-velocities (20) with the accuracy of the sign of the signature is equal to the norm of the Einstein subtraction (4):

$$
\|\mu \triangleleft \nu\|^{2}=-\left|\boldsymbol{u} \ominus_{0} \boldsymbol{v}\right|^{2} .
$$

Proof. The square of the binary four-vector is

$$
\omega^{\mu} \omega_{\mu}=\frac{c^{6}}{(\mu \circ \nu)^{2}}-c^{2} .
$$


The easiest way to calculate the square of the subtraction of velocity is in a properly directed coordinate system $x y z$ :

$$
\begin{gathered}
u_{x}^{\prime 2}+u_{y}^{\prime 2}=\frac{\left(u_{x}-v\right)^{2}+u_{y}^{2}\left(1-v^{2} / c^{2}\right)}{\left(1-u_{x} v / c^{2}\right)^{2}}= \\
\frac{c^{2}\left(1-u_{x} v / c^{2}\right)^{2}-\left(c^{2}-u^{2}\right)\left(1-v^{2} / c^{2}\right)}{\left(1-u_{x} v / c^{2}\right)^{2}}
\end{gathered}
$$

which with the opposite sign equals (22), Q.E.D.

Similarly to the dependence of the four-velocity on the three-velocity (9) or more exact the four-force on the three-force, we can introduce a three-dimensional equivalent for the binary four-velocity

$$
\omega^{\mu}=\left(\gamma_{v} \boldsymbol{w} \boldsymbol{v} / c, \gamma_{v} \boldsymbol{w}\right) .
$$

The three-dimensional relative binary velocity [22] (jet velocity [19]) can be expressed as follows:

$$
\boldsymbol{w}=\frac{\boldsymbol{u}-\boldsymbol{v}+(\boldsymbol{u} \times \boldsymbol{v}) \times \boldsymbol{v} / c^{2}}{1-\boldsymbol{u} \boldsymbol{v} / c^{2}}=: \boldsymbol{u} \ominus_{\perp} \boldsymbol{v} .
$$

At the same time, a new three-velocity subtraction operation was introduced that is simpler than operations $\ominus_{0}$ and $\ominus_{\|}$.

Statement 3. Binary 3D subtraction of velocity vectors (25) is of the form

$$
\boldsymbol{u} \ominus_{\perp} \boldsymbol{v}=\boldsymbol{w}=\psi \boldsymbol{u}-\boldsymbol{v},
$$

where the function $\psi$ results from the parallel subtraction according to (11).

Proof. Using (11) with (26) allows to calculate

$$
\psi=\frac{1-v^{2} / c^{2}}{1-u_{x} v / c^{2}}=\frac{1-v^{2} / c^{2}}{1-\boldsymbol{u v} / c^{2}}
$$

which inserted into (26) after simplifications leads to 3D binary subtraction (25), Q.E.D.

The above statement shows that $3 \mathrm{D}$ vector subtraction differs from the Einstein subtraction in orthogonal component

$$
w_{y}=\left(\boldsymbol{u} \ominus_{\perp} \boldsymbol{v}\right)_{y}=u_{y} \frac{1-v^{2} / c^{2}}{1-u_{x} v / c^{2}} .
$$

Seemingly, the difference relative to (3) is not large and does not seem to solve the non-intuitive problem (apart from time dilation) of scaling the perpendicular component. However, the problem of this scaling disappears if the subtracted velocities have equal components $u_{x}=v \equiv v_{x}$.

The above property is important in the computation of the 3D binary velocity differential (differential of jet velocity):

$$
\begin{aligned}
\mathrm{d} w_{x} & :=\left.\frac{\partial w_{x}}{\partial u_{x}}\right|_{u_{x}=v} \mathrm{~d} u_{x}=\gamma_{v}^{2} \mathrm{~d} u_{x} \\
\mathrm{~d} w_{y} & :=\left.\frac{\partial w_{y}}{\partial u_{x}}\right|_{u_{y}=0} \mathrm{~d} u_{x}+\left.\frac{\partial w_{y}}{\partial u_{y}}\right|_{u_{x}=v} \mathrm{~d} u_{y}=\mathrm{d} u_{y} .
\end{aligned}
$$

Relative 3D binary velocity differentials coincide with axial velocity differentials as opposed to the Einstein subtraction differentials.

\section{Ternary subtraction of $4 \mathrm{D}$ and $3 \mathrm{D}$ velocities}

Based on (18) and (20), we already know that naive subtraction of the four-velocities (10) has little specific sense. However, we can consider such a subtraction projected onto a 3D hypersurface orthogonal to a certain reference four-velocity $\sigma^{\mu}$ :

$$
\xi_{(\sigma)}^{\mu}:=\left(\mu \triangle_{\sigma} \nu\right)^{\mu}:=\lambda P_{\perp \beta}^{\mu}(\sigma)\left(\mu^{\beta}-\nu^{\beta}\right) .
$$

It is therefore a ternary velocity subtraction dependent on the three four-velocities. The orthogonal projection operator has the form $P_{\perp \beta}^{\mu}=\delta_{\beta}^{\mu}-$ $\sigma^{\mu} \sigma_{\beta} / c^{2}$. In order to calculate the form factor $\lambda$ for the sake of simplicity, we will limit ourselves to the three-dimensional approach, assuming that $\sigma^{\mu}=\left(c^{2}, 0,0,0\right)$. This leads to a $3 \mathrm{D}$ relative ternary velocity

$$
\boldsymbol{W}:=\boldsymbol{u} \ominus \wedge \boldsymbol{v}:=\lambda\left(\gamma_{u} \boldsymbol{u}-\gamma_{v} \boldsymbol{v}\right) .
$$

The ternarity of this operation is implicitly hidden in choice $\sigma$. Regardless of the choice of $\lambda$, this operation is not equal to any of the previously discussed. Nevertheless, in the case of axial (parallel vectors), a condition can be imposed on $\lambda$ conforming to standard subtraction

$$
u \ominus_{\wedge} v:=u \ominus v \quad(\boldsymbol{u} \| \boldsymbol{v})
$$

Unfortunately, the condition cannot be extended to each case for a parallel component. The given definition condition allows, however, to calculate

$$
\begin{aligned}
\lambda & =\frac{u-v}{\left(1-\frac{u v}{c^{2}}\right)\left(\gamma_{u} u-\gamma_{v} v\right)}= \\
& \frac{(u-v)\left(\gamma_{u}^{-1}+\gamma_{v}^{-1}\right)}{\left(1-\frac{u v}{c^{2}}\right)\left(u-v+\frac{\gamma_{u}}{\gamma_{v}} u-\frac{\gamma_{v}}{\gamma_{u}} v\right)}= \\
& \frac{\gamma_{u}^{-1}+\gamma_{v}^{-1}}{\left(1-\frac{u v}{c^{2}}\right)\left(1+\gamma_{u} \gamma_{v}\left(1+\frac{u v}{c^{2}}\right)\right)}= \\
& \frac{\gamma_{u}^{-1}+\gamma_{v}^{-1}}{1-\frac{u v}{c^{2}}+\gamma_{u} \gamma_{v}\left(1-\frac{u^{2} v^{2}}{c^{2}}\right)} .
\end{aligned}
$$

We will now make a generalization of this expression for the case of any velocities, assuming that the square of the velocity concerns the square of the norm $\left(u^{2}=\boldsymbol{u}^{2}\right)$ and the expression of the first degree in a given velocity denotes its component $\left(u=u_{x}, u v=u_{x} v=\boldsymbol{u v}\right)$. The validity of these assumptions will be further confirmed in the form of the lemma proof. Applying $u^{2} / c^{2}=1-\gamma_{u}^{-2}$, we get

$$
\begin{gathered}
\lambda=\frac{\gamma_{u}^{-1}+\gamma_{v}^{-1}}{1-\frac{\boldsymbol{u} \boldsymbol{v}}{c^{2}}+\gamma_{u} \gamma_{v}\left(1-\frac{\boldsymbol{u}^{2} \boldsymbol{v}^{2}}{c^{4}}\right)}= \\
\frac{\gamma_{u}+\gamma_{v}}{\gamma_{u} \gamma_{v}\left(1-\frac{\boldsymbol{u} \boldsymbol{v}}{c^{2}}\right)+\gamma_{u}^{2}+\gamma_{v}^{2}-1} .
\end{gathered}
$$

The covariant equivalent of this expression is

$$
\lambda=\frac{c^{2} \sigma \circ \mu+c^{2} \sigma \circ \nu}{c^{2} \mu \circ \nu+(\sigma \circ \mu)^{2}+(\sigma \circ \nu)^{2}-c^{4}} .
$$


Finally, the relative ternary four-velocity becomes

$$
\begin{aligned}
\xi_{(\sigma)}^{\mu} & =\left(\mu \triangle_{\sigma} \nu\right)^{\mu}= \\
& \frac{\sigma \circ(\mu+\nu)\left(c^{2} \delta_{\beta}^{\mu}-\sigma^{\mu} \sigma_{\beta}\right)\left(\mu^{\beta}-\nu^{\beta}\right)}{(\sigma \circ \mu)^{2}+(\sigma \circ \nu)^{2}+c^{2} \mu \circ \nu-c^{4}} .
\end{aligned}
$$

This velocity, written differently and derived differently, was first published by Oziewicz in 2004 [17-19]. Ternary subtraction is a generalization of binary subtraction which is a special case $(\sigma=\nu)$ :

$$
\mu \triangleleft \nu=\mu \triangle_{\nu} \nu .
$$

Another special case $\left(\sigma_{i}=0\right)$ is the already introduced three-dimensional ternary relative velocity

$$
\boldsymbol{W}=\boldsymbol{u} \ominus_{\wedge} \boldsymbol{v}=\frac{\left(\gamma_{u}+\gamma_{v}\right)\left(\gamma_{u} \boldsymbol{u}-\gamma_{v} \boldsymbol{v}\right)}{\gamma_{u} \gamma_{v}\left(1-\frac{\boldsymbol{u} \boldsymbol{v}}{c^{2}}\right)+\gamma_{u}^{2}+\gamma_{v}^{2}-1} .
$$

This velocity, written and derived somewhat differently, first appeared in 2012 in the Dragan [23] lectures notes, but has not been published so far either in the form of an article or in the form of a preprint (except for the Polish lectures). The poster [22] provides a visualized interpretation of the 3D ternary velocity using the so-called laufer/bishop method. Both 4D and 3D ternary subtractions are antisymmetric

$$
\begin{aligned}
& \mu \triangle_{\sigma} \nu=-\nu \triangle_{\sigma} \mu, \\
& \boldsymbol{u} \ominus_{\wedge} \boldsymbol{v}=-\boldsymbol{v} \ominus_{\wedge} \boldsymbol{u} .
\end{aligned}
$$

Other than the one-dimensional velocity subtractions considered in this paper do not have this property - including the Einstein subtraction. The differences do not end there.

Statement 4. Ternary 3D subtraction, with the exception of parallel velocities, does not generally coincide with the Einstein subtraction in the subtrahend direction, nor do the norms of the results of these operations

$$
\begin{aligned}
& \left(\boldsymbol{u} \ominus_{\wedge} \boldsymbol{v}\right)_{\boldsymbol{v}} \not \equiv\left(\boldsymbol{u} \ominus_{0} \boldsymbol{v}\right)_{\boldsymbol{v}}, \\
& \left|\boldsymbol{u} \ominus_{\wedge} \boldsymbol{v}\right| \not \equiv\left|\boldsymbol{u} \ominus_{0} \boldsymbol{v}\right| .
\end{aligned}
$$

Proof. It is enough to indicate one example for which the considered equations do not exist. So let us consider perpendicular velocities with equal values: $u=v=v_{x}=u_{y}=0.6 c$. Einstein's subtracting leads to the components of the velocity vector

$$
\begin{aligned}
& u_{x}^{\prime}=\frac{0-0.6 c}{1-0 \times 0.6}=-0.6 c, \\
& u_{y}^{\prime}=0.6 c \frac{\sqrt{1-0.6^{2}}}{1-0 \times 0.6}=0.48 c,
\end{aligned}
$$

whose value is $u^{\prime} \approx 0.7684 c$. Whereas the components of the 3D ternary velocity are

$$
\begin{aligned}
& W_{x}=\frac{\left(\frac{5}{4}+\frac{5}{4}\right) \times\left(\frac{5}{4} \times 0-\frac{5}{4} \times \frac{3}{5} c\right)}{\frac{5}{4} \times \frac{5}{4}(1-0)+\frac{5^{2}}{4^{2}}+\frac{5^{2}}{4^{2}}-1}= \\
& -\frac{30}{59} c \approx-0.5085 c,
\end{aligned}
$$

$$
\begin{aligned}
& W_{y}=\frac{\left(\frac{5}{4}+\frac{5}{4}\right)\left(\frac{5}{4} \times \frac{3}{5} c-\frac{5}{4} \times 0\right)}{\frac{5}{4} \times \frac{5}{4}(1-0)+\frac{5^{2}}{4^{2}}+\frac{5^{2}}{4^{2}}-1}= \\
& \frac{30}{59} c \approx 0.5085 c,
\end{aligned}
$$

which gives it value $W \approx 0.7191 c$. Thus, neither the $x$ components nor the values of the considered velocities are equal, Q.E.D.

Statement 4 would suggest that the 3D ternary velocity is weakly anchored in the Lorentz transformation. However, nothing could be more wrong, as shown below (see [23]).

Lemma. The Lorentz boost (the Einstein subtraction) with a ternary $3 D$ velocity for a velocity, which is minuend ternary subtraction, restores ternary subtrahend velocity

$$
\boldsymbol{u} \ominus_{0} \boldsymbol{W}=\boldsymbol{u} \ominus_{0}\left(\boldsymbol{u} \ominus_{\wedge} \boldsymbol{v}\right)=\boldsymbol{v} .
$$

In other words, in the above sense, $3 D$ ternary subtraction is a left-inverse operation of the Einstein subtraction.

Proof. Proving the lemma thesis is computationally complicated and it cannot be done just by boost in the $x$ direction. A significant difficulty is calculating the Lorentz factor for velocity $\boldsymbol{W}$ :

$$
\frac{1}{\gamma_{W}^{2}}=1-\frac{W^{2}}{c^{2}}=\frac{\left(\gamma_{u} \gamma_{v} \frac{u v}{c^{2}}+\gamma_{u} \gamma_{v}+1\right)^{2}}{\left(\gamma_{u} \gamma_{v}\left(1-\frac{u v}{c^{2}}\right)+\gamma_{u}^{2}+\gamma_{v}^{2}-1\right)^{2}},
$$

which can be written more compactly

$$
\frac{1}{\gamma_{W}}+1=\left(\gamma_{u}+\gamma_{v}\right) \lambda
$$

The main calculations can now be made

$$
\begin{gathered}
\boldsymbol{u} \ominus_{0} \boldsymbol{W}=\frac{\frac{1}{\gamma_{W}} \boldsymbol{u}-\boldsymbol{W}+\frac{\boldsymbol{u} \boldsymbol{W} / c^{2}}{1+\gamma_{W}^{-1}} \boldsymbol{W}}{1-\frac{\boldsymbol{u} \boldsymbol{W}}{c^{2}}}= \\
\frac{B \boldsymbol{u}+C \boldsymbol{v}}{1-\frac{\boldsymbol{u} \boldsymbol{W}}{c^{2}}} .
\end{gathered}
$$

Now it is enough to calculate the coefficients $B$ and $C$ :

$$
\begin{aligned}
& B=-1+\left(\gamma_{v}+\frac{\gamma_{u}^{2}-1-\gamma_{u} \gamma_{v} \frac{\boldsymbol{u} \boldsymbol{v}}{c^{2}}}{\gamma_{u}+\gamma_{v}}\right) \lambda=0, \\
& C=\frac{\gamma_{v}\left(\gamma_{u} \gamma_{v}+1+\gamma_{u} \gamma_{v} \frac{\boldsymbol{u v}}{c^{2}}\right)}{\gamma_{u}\left(\gamma_{u}+\gamma_{v}\right)} \lambda=1-\frac{\boldsymbol{u} \boldsymbol{W}}{c^{2}} .
\end{aligned}
$$

Based on these calculations, the value of the expression (49) is $\boldsymbol{v}$, Q.E.D.

The ordinarily understood inverse Lorentz transformation is the right-hand inverse (according to the adopted notation convention):

$$
\boldsymbol{u} \ominus_{0} \boldsymbol{v}=\boldsymbol{u}^{\prime} \rightarrow \boldsymbol{u}=\boldsymbol{u}^{\prime} \ominus_{0}(-\boldsymbol{v}) .
$$

On the other hand, the ternary operation can be understood as a left-inverse operation

$$
\boldsymbol{u} \ominus_{0} \boldsymbol{W}=\boldsymbol{v} \rightarrow \boldsymbol{W}=\boldsymbol{u} \ominus_{\wedge} \boldsymbol{v} .
$$

Like the Einstein subtraction, left-hand application is the left-hand inverse of ternary action (46). It is worth writing it explicitly in both orders

$$
\boldsymbol{u} \ominus_{0}\left(\boldsymbol{u} \ominus_{\wedge} \boldsymbol{v}\right)=\boldsymbol{u} \ominus_{\wedge}\left(\boldsymbol{u} \ominus_{0} \boldsymbol{v}\right)=\boldsymbol{v} .
$$


To sum up, the main reason for the difference between the 3D ternary velocity subtraction from the Einstein subtraction is the noncommutativity of velocity composition (and the Lorentz group). The impact of the lack of associative velocity composition is not directly apparent here, and the Lorentz group is associative by definition.

The differential of the 3D ternary velocity, which is crucial for this paper, remains to be calculated. The ternary velocity is so homogeneous due to its components that we can immediately calculate the vector differential

$$
\begin{gathered}
\mathrm{d} \boldsymbol{W}:=\left.\frac{\partial \boldsymbol{W}}{\partial u_{i}}\right|_{\boldsymbol{u}=\boldsymbol{v}} \mathrm{d} u_{i}=\mathrm{d} \boldsymbol{u}+\frac{\gamma_{v}^{2}}{c^{2}} \boldsymbol{v}(\boldsymbol{v} \mathrm{d} \boldsymbol{u})= \\
\mathrm{d} \boldsymbol{u}_{\perp}+\gamma_{v}^{2} \mathrm{~d} \boldsymbol{u}_{\|} .
\end{gathered}
$$

As before, the velocity differential $\boldsymbol{v}$ would only differ in sign and names of symbols. Thus, as can be seen, the 3D ternary velocity differential is consistent with the $3 \mathrm{D}$ axial velocity differential and the 3D binary velocity differential. This compatibility is not accidental and reflects a class of differentially equivalent operations to which the Einstein subtraction does not belong.

\section{Relativistic $4 \mathrm{D}$ and $3 \mathrm{D}$ acceleration}

We will start with the 4D approach as it will pave the way for the main original $3 \mathrm{D}$ result. The standard four-acceleration is derivative of the fourvelocity respect to self-time [16]:

$$
\begin{aligned}
& \alpha^{\mu}=\frac{\mathrm{d} \mu^{\mu}}{\mathrm{d} \tau}=\left(\alpha^{0}, \vec{\alpha}\right)= \\
& \quad\left(\gamma_{u}^{4} \boldsymbol{a u} / c, \gamma_{u}^{2} \boldsymbol{a}+\gamma_{u}^{4}(\boldsymbol{a u}) \boldsymbol{u} / c^{2}\right) .
\end{aligned}
$$

Despite the simple definition, four-acceleration depends on the ordinary three-acceleration $\boldsymbol{a}$ in a very complicated way. The same result can be obtained for a binary four-velocity if we identify the subtracted four-velocities after derivative calculated

$$
\alpha^{\mu}=\left.\frac{\mathrm{d} \omega^{\mu}}{\mathrm{d} \tau}\left(\tau_{0}, \tau\right)\right|_{\tau=\tau_{0}}=\left.\frac{\mathrm{d}\left(\mu(\tau) \varangle \mu\left(\tau_{0}\right)\right)^{\mu}}{\mathrm{d} \tau}\right|_{\tau=\tau_{0}} .
$$

This is equivalent to writing the derivative definition, in which the ordinary subtraction is replaced by a binary $4 \mathrm{D}$ subtraction

$$
\alpha^{\mu}=\lim _{\Delta \tau \rightarrow 0} \frac{\left(\mu\left(\tau_{0}+\Delta \tau\right) \varangle \mu\left(\tau_{0}\right)\right)^{\mu}}{\Delta \tau} .
$$

Thanks to this notation, it is clear that when calculating the acceleration (or differential) from the relative velocity, the subtracted velocities $\nu^{\mu}$ and $\mu^{\mu}$ should be tending to each other.

For the ternary four-velocity, there is also the third reference four-velocity $\sigma^{\mu}$. If this four-velocity were to take the same value as the previous two, then the four-acceleration calculations would coincide with the binary calculations. Thus, it is worth treating $\sigma^{\mu}$ as the four-velocity of the independent selected frame of reference, in which the moving time will be measured and the acceleration calculated. Time in the inertial frame $\sigma$ relative to selftime of body with a four-velocity $\mu^{\mu}(\tau)$ is described by time dilation $\mathrm{d} T=\left(\sigma \circ \mu / c^{2}\right) \mathrm{d} \tau=\mathrm{d} \tau / \lambda(\sigma, \mu, \mu)$. This leads to the definition of the four-acceleration from 4D ternary subtraction:

$$
\begin{gathered}
\mathcal{A}_{(\sigma)}^{\mu}:=\frac{\mathrm{d} \xi_{(\sigma)}^{\mu}}{\mathrm{d} T}:=\lim _{\Delta \tau \rightarrow 0} \frac{\left(\mu\left(\tau_{0}+\Delta \tau\right) \Delta_{\sigma} \mu\left(\tau_{0}\right)\right)^{\mu}}{\Delta \tau / \lambda}= \\
\frac{c^{4}}{(\sigma \circ \mu)^{2}}\left(\alpha^{\mu}-(\sigma \circ \alpha) \sigma^{\mu} / c^{2}\right) .
\end{gathered}
$$

The ternary four-acceleration is thus a properly normalized projection of the ordinary four-acceleration. For $\sigma=\mu$ four-acceleration $\mathcal{A}_{(\sigma)}^{\mu}$ coincides with four-acceleration $\alpha^{\mu}$, and for $\sigma_{i}=0$ it amounts to a three-dimensional relativistic acceleration $\boldsymbol{A}$ in the same way that the $4 \mathrm{D}$ ternary velocity comes down to the $3 \mathrm{D}$ ternary velocity.

It is time to define the $3 \mathrm{D}$ relativistic acceleration. For definition, we need a velocity differential based on the difference of velocities, that is on relative velocity. Apart from the ordinary subtraction, we have the Einstein subtraction and three other methods of 3D subtraction: axial, binary, and ternary. The Einstein subtraction leads to a velocity differential in the system of instantaneous rest of the body, which only allows the determination of the rest acceleration $\boldsymbol{a}_{0}=\mathrm{d} \boldsymbol{u}^{\prime} / \mathrm{d} \tau$ [19]. The rest acceleration is not an acceleration per se, so they remain consistent differentials of axial, 3D binary, and ternary velocities. This velocity differential will be called the relativistic differential (of velocity) and will be denoted as follows:

$$
\mathrm{D} \boldsymbol{u}:=\mathrm{d} \boldsymbol{w}_{\mid}=\mathrm{d} \boldsymbol{w}=\mathrm{d} \boldsymbol{W}=\mathrm{d} \boldsymbol{u}_{\perp}+\gamma_{u}^{2} \mathrm{~d} \boldsymbol{u}_{\|} .
$$

Statement 5. The velocity relativistic differentiation operation is a differentiation operation multiplied on both sides by the gamma factor and its inverse (on the left):

$$
\mathrm{D}=\gamma^{-1} \times \mathrm{d} \times \gamma .
$$

$$
\begin{aligned}
& \text { Proof: } \\
& \qquad \mathrm{D} \boldsymbol{u}=\gamma_{u}^{-1} \mathrm{~d}\left(\gamma_{u} \boldsymbol{u}\right)=\mathrm{d} \boldsymbol{u}+\gamma_{u}^{2} \boldsymbol{u}(\boldsymbol{u} \mathrm{d} \boldsymbol{u}) / c^{2},
\end{aligned}
$$

which is equal to (60) by virtue of (55), Q.E.D.

The key relativistic 3D acceleration for this paper can now be defined:

$$
\boldsymbol{A}:=\frac{\mathrm{D} \boldsymbol{u}}{\mathrm{d} t}:=\lim _{\Delta t \rightarrow 0} \frac{\boldsymbol{u}(t+\Delta t) \ominus_{\wedge} \boldsymbol{u}(t)}{\Delta t},
$$

where antisymmetric ternary subtraction can be replaced by binary or axial subtraction. This definition leads to the formula:

$$
\boldsymbol{A}=\boldsymbol{a}+\gamma_{u}^{2} \boldsymbol{u}(\boldsymbol{u} \boldsymbol{a}) / c^{2}=\boldsymbol{a}_{\perp}+\gamma_{u}^{2} \boldsymbol{a}_{\|},
$$

where $\boldsymbol{a}=\mathrm{d} \boldsymbol{u} / \mathrm{d} t$ is the ordinary acceleration.

Four-acceleration can now be expressed simply by acceleration $\boldsymbol{A}$ :

$$
\alpha^{\mu}=\left(\alpha^{0}, \vec{\alpha}\right)=\left(\gamma_{u}^{2} \boldsymbol{A u} / c, \gamma_{u}^{2} \boldsymbol{A}\right) .
$$




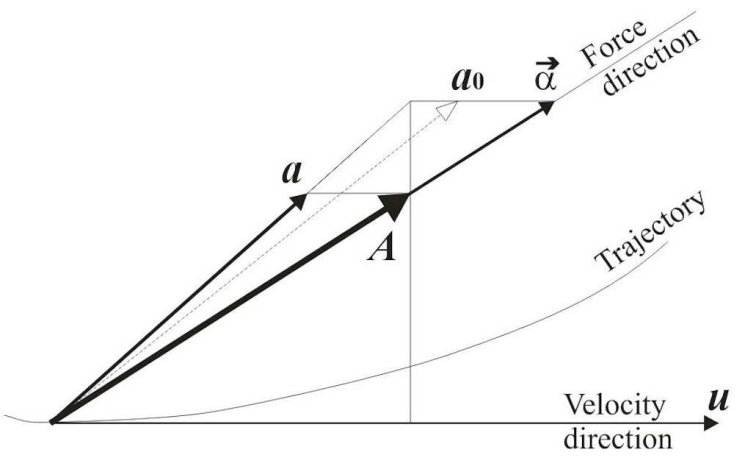

Fig. 1. Diagram showing the relations of the main $3 \mathrm{D}$ acceleration vectors. The new relativistic acceleration $\boldsymbol{A}$ is a parallel projection of ordinary acceleration $\boldsymbol{a}$ along the velocity on the direction of force (or spatial part $\vec{\alpha}$ of the four-acceleration $\alpha^{\mu}$ ). Thanks to this, the construction of acceleration $\boldsymbol{A}$ is simpler than the construction of rest acceleration $\boldsymbol{a}_{0}$.

Thanks to this, also the ternary four-acceleration $\mathcal{A}_{(\sigma)}^{\mu}$ can be expressed as acceleration $\boldsymbol{A}$. However, this is a more complex expression that simplifies for $\sigma_{i}=0$ :

$$
\mathcal{A}_{\left(\sigma_{i}=0\right)}^{\mu}=(0, \boldsymbol{A}) \text {. }
$$

A graphical interpretation of $(64)-(66)$ is shown in Fig. 1.

\section{Conclusion}

It has been shown and proved through the paper that within the Lorentz group it is possible to reasonably subtract velocities in a different way than it was established in SR. First of all, it can be done in an antisymmetric way or in a four-vector way. For example, the antisymmetric operation is the left-hand reciprocal of the Lorentz boost. Velocity subtraction operations made it possible to define different $4 \mathrm{D}$ and $3 \mathrm{D}$ relative velocities. Due to the differential equivalence of these subtraction operations, unambiguous and original 3D relativistic acceleration was introduced. This relativistic acceleration is important for simplifying the dynamics of the SR [19, 22]. Whereas in this article, the relativistic acceleration has been generalized to the $4 \mathrm{D}$ ternary four-acceleration.

\section{References}

[1] W. Rindler, Relativity: Special, General and Cosmological, Oxford University Press, 2007.

[2] A. Einstein, Ann. Phys. 17, 891 (1905).

[3] M. Cannoni, arXiv:1605.00569v2 (2016).
[4] V. Fock, The Theory of Space, Time and Gravitation, 1st ed. 1959, 2nd rev. ed., Pergamon Press, 1964.

[5] M. Fernández-Guasti, Proc. SPIE 8121, 812108 (2011).

[6] A.A. Ungar, Results Math. 16, 168 (1989).

[7] K. Rębilas, Apeiron 15, 206 (2008).

[8] A. Dragan, T. Odrzygóźdź, Am. J. Phys. 81, 631 (2013).

[9] J.F. Barrett, "Review of problems of dynamics in the hyperbolic theory of special relativity", in: Proc. Physical Interpretations of Relativity Theory Conf., Imperial Coll., London 2002, PD Publ., Liverpool 2002, p. 17.

[10] K. Rębilas, Am. J. Phys. 78, 294 (2010).

[11] M. Ahmad, M.S. Alam, Int. J. Reciproc. Symm. Theor. Phys. 1, 9 (2014).

[12] Z. Oziewicz, Int. J. Geom. Methods Mod. Phys. 4, 739 (2007).

[13] M. Ahmad, Int. J. Reciproc. Symm. Theor. Phys. 1, 69 (2014).

[14] J. Kocik, arXiv:1910.06785v1 (2019).

[15] H. Minkowski, in: Nachrichten von der Gesellschaft der Wissenschaften zu Göttingen, Mathematisch-Physikalische Klasse, Berlin 1908, p. 53 (in German).

[16] A.K. Wróblewski, J.A. Zakrzewski, Introduction to Physics, Vol. 1, PWN, Warsaw 1976, (in Polish).

[17] Z. Oziewicz, in: Group Theoretical Methods in Physics, Conference Series, Vol. 185, CRC Press, 2004, p. 439.

[18] Z. Oziewicz, arXiv:1104.0682v1 (2011).

[19] G.M. Koczan, Res. Phys. 24, 104121 (2021).

[20] K. Rębilas, Europ. J. Phys. 34, L55 (2013).

[21] V.J. Bolós, Commun. Math. Phys. 273 , 217 (2007).

[22] G.M. Koczan, poster for XLVI Extraordinary Congress of Polish Physicists on the 100 Years of the Polish Physical Society, Warsaw, 2020.

[23] A. Dragan, monograph - lecture notes, Unusually special theory of relativity, Ch. 3. Thomas-Wigner rotation (2012) (in Polish). 